KUNS-1857

hep-th/0404121

\title{
Cosmological Constant Probing Shape Moduli through Large Extra Dimensions
}

\author{
Satoshi Matsuda ${ }^{\dagger}$ and Shigenori Seki ${ }^{\ddagger *}$ \\ Department of Physics, Graduate School of Science \\ Kyoto University, Kyoto 606, Japan
}

\begin{abstract}
We consider compactification of extra dimensions and numerically calculate Casimir energy which is provided by the mass of Kaluza-Klein modes. For the extra space we consider a torus with shape moduli and show that the corresponding vacuum energy is represented as a function of the moduli parameter of the extra dimensions. By assuming that the Casimir energy may be identified with cosmological constant, we evaluate the size of extra dimensions in terms of the recent data given by the Wilkinson Microwave Anisotropy Probe (WMAP) measurement and the supernovae observations. We suggest that the observed cosmological constant may probe the shape moduli of the extra space by the study of the Casimir energy of the compactified extra dimensions.
\end{abstract}

$\dagger$ matsuda@yukawa.kyoto-u.ac.jp

$\ddagger$ seki@gauge.scphys.kyoto-u.ac.jp

* Moved from Theoretical Physics Laboratory, The Institute of Physical and Chemical Research (RIKEN), Wako 351, Japan on April 1, 2004. 


\section{Introduction}

Extra dimensions have been introduced in order to solve the hierarchy problem [1], and a lot of related works including the milestone papers [1 4 and later developments [5 9] have been done. In these approaches, gauge fields are localized on a four dimensional brane, which is regarded as our real world, and only a graviton can propagate in the extra space, which is transverse to the brane. When the extra space is compact, there exist the Kaluza-Klein modes of the graviton. They contribute to Casimir energy on the four dimensional brane.

On the other hand, the origin of cosmological constant is also a standing problem. Recently the supernovae [10,11] and WMAP [12,13] observations have revealed some parameters of our universe. These new results encourage not only the researchers of cosmology but also the particle physicists [14 [16] toward challenging the resolution of the unsolved problems again. If we assume that the Casimir energy, or in other words the vacuum energy, can be regarded as the cosmological constant, we can estimate the size of extra dimensions in terms of the observational value of the cosmological constant [16].

When we calculate the vacuum energy, we have to perform a summation over the infinite number of Kaluza-Klein modes. In this paper, we introduce a new mathematical technique to achieve this infinite summation, which provides a reasonable regularization and makes it possible to calculate numerically the vacuum energy of all Kaluza-Klein modes.

In [1,2], it is shown that the number of extra dimensions should be two from the consistency with the hierarchy problem. The choice of the two extra dimensions are also favourable from the viewpoint of the cosmological constant problem [16]. So we consider the extra space of two dimensions in this paper. In particular we concentrate on a torus with shape moduli. The compactification on the torus and the Kaluza-Klein masses under a standard mass operator are analysed in [17 22]. In this paper we additionally impose

$\left(\mathbb{Z}_{2}\right)^{2}$ symmetry or Dirichlet conditions on the torus. Since the standard mass operator is inconsistent with these boundary conditions, we propose a new mass operator incorporating the shape moduli in consistency with the boundary conditions on the torus. Using this operator, we calculate the vacuum energy. The resulting expression depends on the shape moduli parameter, as a function of which the structure of the extra space can be probed by confronting the numerical values of the Casimir energy with the observed cosmological constant [10]13]. 
The paper is organized as follows. In the section 2 , we consider the $\left(\mathbb{Z}_{2}\right)^{2}$ symmetry and Dirichlet conditions on the torus with the shape moduli. Requiring the invariance under these conditions, we introduce the new mass operator with the shape moduli. In the section 3, we show the technical details of the regularization of the vacuum energy to which the infinite number of Kaluza-Klein modes contribute. We also present the numerical results of the calculation of the vacuum energy. In the section 4 , we identify these numerical values of the vacuum energy with the cosmological constant which is given by [10 13] and evaluate the size of extra dimensions. The section 5 is devoted to conclusions and discussion.

\section{Shape Moduli and Mass Operator}

Though various kinds of extra space are available, we consider a two dimensional torus, $T^{2}$, in this paper. In the covering space of $T^{2}$, we require in the standard way the two periodic conditions,

$$
\begin{aligned}
& \left(y_{1}, y_{2}\right) \sim\left(y_{1}+2 \pi R_{1}, y_{2}\right), \\
& \left(y_{1}, y_{2}\right) \sim\left(y_{1}+2 \pi R_{2} \cos \theta, y_{2}+2 \pi R_{2} \sin \theta\right) .
\end{aligned}
$$

$R_{1}$ and $R_{2}$ denote the radii of two cycles, while $\theta$ parametrizes the shape moduli of $T^{2}$. After the Kaluze-Klein compactification on such a torus, the eigen functions for the usual $(\text { mass })^{2}$ operator $-\left[\left(\partial / \partial y_{1}\right)^{2}+\left(\partial / \partial y_{2}\right)^{2}\right]$ are proportional to

$$
\exp \left[i \frac{n_{1}}{R_{1}}\left(y_{1}-\frac{y_{2}}{\tan \theta}\right)+i \frac{n_{2}}{R_{2}} \frac{y_{2}}{\sin \theta}\right]
$$

where $n_{1}, n_{2} \in \mathbb{Z}$, and the eigen values [18 20] become $n_{1}^{2} / R_{1}^{2}+n_{2}^{2} / R_{2}^{2}-2 n_{1} n_{2} \cos \theta /\left(R_{1} R_{2}\right)$. For these mass eigen values, we can numerically calculate, in the same way as in [16], the vacuum energy in which we have to sum up all the Kaluza-Klein modes.

Now we introduce new coordinates $\left(X_{1}, X_{2}\right)$ defined by

$$
X_{1} \equiv \frac{y_{1}}{R_{1}}-\frac{y_{2}}{R_{1} \tan \theta}, \quad X_{2} \equiv \frac{y_{2}}{R_{2} \sin \theta} .
$$

The periodic condition (2.1) is rewritten as

$$
\begin{aligned}
& \left(X_{1}, X_{2}\right) \sim\left(X_{1}+2 \pi, X_{2}\right), \\
& \left(X_{1}, X_{2}\right) \sim\left(X_{1}, X_{2}+2 \pi\right) .
\end{aligned}
$$


The eigen functions (2.2) are equal to $\exp \left(i n_{1} X_{1}+i n_{2} X_{2}\right)$ with $n_{i} \in \mathbb{Z}(i=1,2)$.

In addition, let us impose $\left(\mathbb{Z}_{2}\right)^{2}$ symmetry on $T^{2}$.

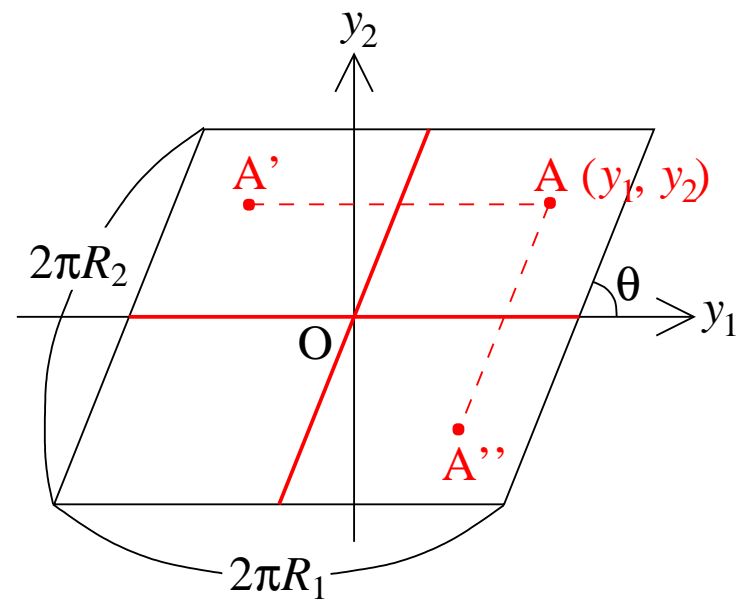

fig. 1: A fundamental region of $T^{2}$ in the covering space.

This symmetry is realized by the two mirror symmetry along the two thick lines represented in fig. 1 . In terms of the coordinates $\left(y_{1}, y_{2}\right)$, the symmetry is denoted by the following identification

$$
\begin{aligned}
& \left(y_{1}, y_{2}\right) \sim\left(-y_{1}+\frac{2 y_{2}}{\tan \theta}, y_{2}\right), \\
& \left(y_{1}, y_{2}\right) \sim\left(y_{1}-\frac{2 y_{2}}{\tan \theta},-y_{2}\right) .
\end{aligned}
$$

In fig. 1, the point $\mathrm{A}^{\prime}\left(-y_{1}+\frac{2 y_{2}}{\tan \theta}, y_{2}\right)$ is identified with the point $\mathrm{A}\left(y_{1}, y_{2}\right)$ by one $\mathbb{Z}_{2}$ symmetry and the point A" $\left(y_{1}-\frac{2 y_{2}}{\tan \theta},-y_{2}\right)$ is also identified with the point A by the other $\mathbb{Z}_{2}$ symmetry. Since the $\left(\mathbb{Z}_{2}\right)^{2}$ symmetry (2.5) can be interpreted as the reflection symmetry given by

$$
\left(X_{1}, X_{2}\right) \sim\left(-X_{1}, X_{2}\right), \quad\left(X_{1}, X_{2}\right) \sim\left(X_{1},-X_{2}\right),
$$

the wave function under the $\left(\mathbb{Z}_{2}\right)^{2}$ symmetry, through the use of the coordinates $\left(X_{1}, X_{2}\right)$, should be an even function with respect to each variable, $X_{1}$ or $X_{2}$. By the analogy of (2.2), we may suppose that the wave function satisfying (2.1) and (2.5) is $\cos \left(n_{1} X_{1}\right) \cos \left(n_{2} X_{2}\right)$. But this function is not an eigen function of the (mass $)^{2}$ operator $-\left[\left(\partial / \partial y_{1}\right)^{2}+\left(\partial / \partial y_{2}\right)^{2}\right]$.

So we propose a new mass operator which has $\cos \left(n_{1} X_{1}\right) \cos \left(n_{2} X_{2}\right)$ as an eigen function. First we note that the following differential operators simply change signs under the operation of $\left(\mathbb{Z}_{2}\right)^{2}$ symmetry on $T^{2}$, (2.5):

$$
\frac{\partial}{\partial y_{1}}=\frac{1}{R_{1}} \frac{\partial}{\partial X_{1}}, \quad \frac{\partial}{\partial y_{2}}+\frac{1}{\tan \theta} \frac{\partial}{\partial y_{1}}=\frac{1}{R_{2} \sin \theta} \frac{\partial}{\partial X_{2}} .
$$


Though there are a variety of choices for the mass operator, the above observation has led us to consider, as the simplest toy model, the following generic (mass) ${ }^{2}$ operator defined by

$$
-\left[\left(\frac{1}{R_{1}} \frac{\partial}{\partial X_{1}}\right)^{2}+\left(\frac{1}{R_{2} \sin \theta} \frac{\partial}{\partial X_{2}}\right)^{2}\right]=-\left[\frac{1}{\sin ^{2} \theta} \frac{\partial^{2}}{\partial y_{1}^{2}}+\frac{\partial^{2}}{\partial y_{2}^{2}}+\frac{2}{\tan \theta} \frac{\partial}{\partial y_{1}} \frac{\partial}{\partial y_{2}}\right] .
$$

The proposed (mass) $)^{2}$ operator (2.8) is invariant under the $\left(\mathbb{Z}_{2}\right)^{2}$ symmetry (2.5), and reduces to the standard one for $\theta=\pi / 2$, that is, when the fundamental region of $T^{2}$ is rectangular.

In other words, though we can make a variety of choices for the metric in the extra space, the $\left(\mathbb{Z}_{2}\right)^{2}$ symmetry on $T^{2}$ has guided us to choose the simplest case of (2.8) for the mass operator. The corresponding metric of $T^{2}$ can be proved to be

$$
d s^{2}=d y_{1}^{2}-\frac{1}{\tan \theta}\left(d y_{1} d y_{2}+d y_{2} d y_{1}\right)+\frac{1}{\sin ^{2} \theta} d y_{2}^{2} .
$$

This metric is naturally invariant under the $\left(\mathbb{Z}_{2}\right)^{2}$ symmetry (2.5), and again reduces to the standard rectangular one when $\theta=\pi / 2$.

In the next section, we use the mass operator (2.8) and calculate the vacuum energy.

\section{Vacuum Energy and Boundary Conditions}

We consider the torus compactification with shape moduli. The torus $T^{2}$ without shape moduli is the same as $\left(S^{1}\right)^{\otimes 2}$. The vacuum (Casimir) energy which is enhanced by the Kaluza-Klein modes derived from the compactification on the $d$ dimensional extra space $\left(S^{1}\right)^{\otimes d}$ has already been discussed in [16]. In this section we consider the compactification on $T^{2}$ with shape moduli and the generic mass operator (2.8) as has been discussed in the section 2. We then calculate the vacuum energy in our four dimensional space to which all the Kaluza-Klein modes from the extra space contribute.

For simplicity we set $R_{1}=R_{2} \equiv R$.

\section{1. without a boundary condition}

The eigen functions compactified on $T^{2}$ with the mass operator (2.8) are described by $\exp \left(i n_{1} X_{1}\right) \exp \left(i n_{2} X_{2}\right)$ from (2.4), and the masses of Kaluza-Klein modes then become

$$
M=\frac{1}{R} \sqrt{n_{1}^{2}+\frac{n_{2}^{2}}{\sin ^{2} \theta}} \equiv \frac{1}{R} f_{n_{1}, n_{2}}(\theta), \quad n_{1}, n_{2} \in \mathbb{Z} .
$$


We consider the zero point energies of these Kaluze-Klein modes and the vacuum energy of our four dimensional world is represented by

$$
E=\frac{1}{2} \sum_{n_{1}, n_{2}=-\infty}^{\infty} \int \frac{d^{3} k}{(2 \pi)^{3}} \sqrt{\mathbf{k}^{2}+M^{2}}
$$

Note that, if the Kaluza-Klein modes are for gravitons, we have to insert a factor corresponding to the number of polarization degrees of freedom, $(2+2)(3+2) / 2-1=9$, in front of the right hand side of (3.1) [23]. In the following we consider only a scalar degree of freedom for simplicity. Since the integration in (3.1) is generally divergent, we use dimensional regularization for it and obtain

$$
E=-\frac{1}{64 \pi^{2}} \sum_{n_{1}, n_{2}=-\infty}^{\infty} M^{4}\left[\ln \left(\frac{\lambda^{2}}{M^{2}}\right)+\frac{3}{2}\right]
$$

where the parameter $\lambda$ has a mass dimension. Since the infinite summation for $\left(\sqrt{n_{1}^{2}+n_{2}^{2} / \sin ^{2} \theta}\right)^{4}$ is calculated as

$$
\begin{aligned}
\sum_{n_{1}, n_{2}=-\infty}^{\infty} f_{n_{1}, n_{2}}(\theta)^{4} & =\sum_{n, m=-\infty}^{\infty}\left(1+\frac{1}{\sin ^{4} \theta}\right) n^{4}+\sum_{n, m=-\infty}^{\infty} \frac{2}{\sin ^{3} \theta} n^{2} m^{2} \\
& =4\left(1+\frac{1}{\sin ^{4} \theta}\right) \zeta(0) \zeta(-4)+\frac{8}{\sin ^{2} \theta} \zeta(-2)^{2} \\
& =0,
\end{aligned}
$$

where $\zeta(-2)=\zeta(-4)=0$, the vacuum energy $(3.2)$ is reduced to

$$
\begin{aligned}
E & =\frac{1}{32 \pi^{2} R^{4}} \sum_{n_{1}, n_{2}=-\infty}^{\infty} f_{n_{1}, n_{2}}{ }^{4} \ln f_{n_{1}, n_{2}} \\
& =\left.\frac{1}{32 \pi^{2} R^{4}} \sum_{n_{1}, n_{2}=-\infty}^{\infty} \frac{d}{d x} f_{n_{1}, n_{2}}{ }^{x}\right|_{x=4} .
\end{aligned}
$$

In calculating the vacuum energy, the infinite summation shows up and appears to diverge because of the contribution of the infinite number of Kaluza-Klein modes. It is known that in some special cases [24,25] the vacuum energy can be regularized and are calculated exactly in terms of Epstein's zeta function. Since in general we can not use such a 
regularization, we suggest a new numerical method as follows. By the use of the Fourier transformation [26],

$$
\left(\sqrt{\sum_{i=1}^{d} \sigma_{i}^{2}}\right)^{x}=-\frac{2^{x} \sin \frac{\pi x}{2}}{\pi^{\frac{d}{2}+1}} \Gamma\left(1+\frac{x}{2}\right) \Gamma\left(\frac{x+d}{2}\right) \int_{-\infty}^{\infty} \frac{\exp \left(i \sum_{i=1}^{d} \sigma_{i} q_{i}\right)}{\left(\sqrt{\sum_{i=1}^{d} q_{i}^{2}}\right)^{x+d}} d q_{1} \cdots d q_{d}
$$

which is valid for any real values of $\left(\sigma_{1}, \sigma_{2}, \cdots, \sigma_{d}\right)$, we obtain

$$
\begin{aligned}
\sum_{n_{1}, n_{2}=-\infty}^{\infty} f_{n_{1}, n_{2}} x & =\sum_{n_{1}, n_{2}=-\infty}^{\infty}-\frac{2^{x} \sin \frac{\pi x}{2}}{\pi^{2}} \Gamma\left(1+\frac{x}{2}\right)^{2} \int_{-\infty}^{\infty} \frac{\exp \left(i n_{1} q_{1}+i \frac{n_{2}}{\sin \theta} q_{2}\right)}{\left(\sqrt{q_{1}^{2}+q_{2}^{2}}\right)^{x+2}} d q_{1} d q_{2} \\
& =-\frac{\sin \frac{\pi x}{2}}{\pi^{2+x}} \Gamma\left(1+\frac{x}{2}\right)^{2} \sin \theta \sum_{\substack{m_{1}, m_{2}=-\infty \\
\left(m_{1}, m_{2}\right) \neq(0,0)}}^{\infty} \frac{1}{\left(m_{1}^{2}+m_{2}^{2} \sin ^{2} \theta\right)^{\frac{x+2}{2}}},
\end{aligned}
$$

where we have used $\sum_{n=-\infty}^{\infty} \exp (i n q)=2 \pi \sum_{m=-\infty}^{\infty} \delta(q-2 \pi m)$. The contribution of $\left(m_{1}, m_{2}\right)=(0,0)$ mode vanishes, which can be proven by employing a generalized function technique [26]. From (3.3) and (3.5), the vacuum energy becomes

$$
E=-\frac{1}{64 \pi^{7} R^{4}} \Gamma(3)^{2} \sin \theta \sum_{\substack{m_{1}, m_{2}=-\infty \\\left(m_{1}, m_{2}\right) \neq(0,0)}}^{\infty} \frac{1}{\left(m_{1}^{2}+m_{2}^{2} \sin ^{2} \theta\right)^{3}} .
$$

Since (3.6) is convergent, next we numerically calculate it.

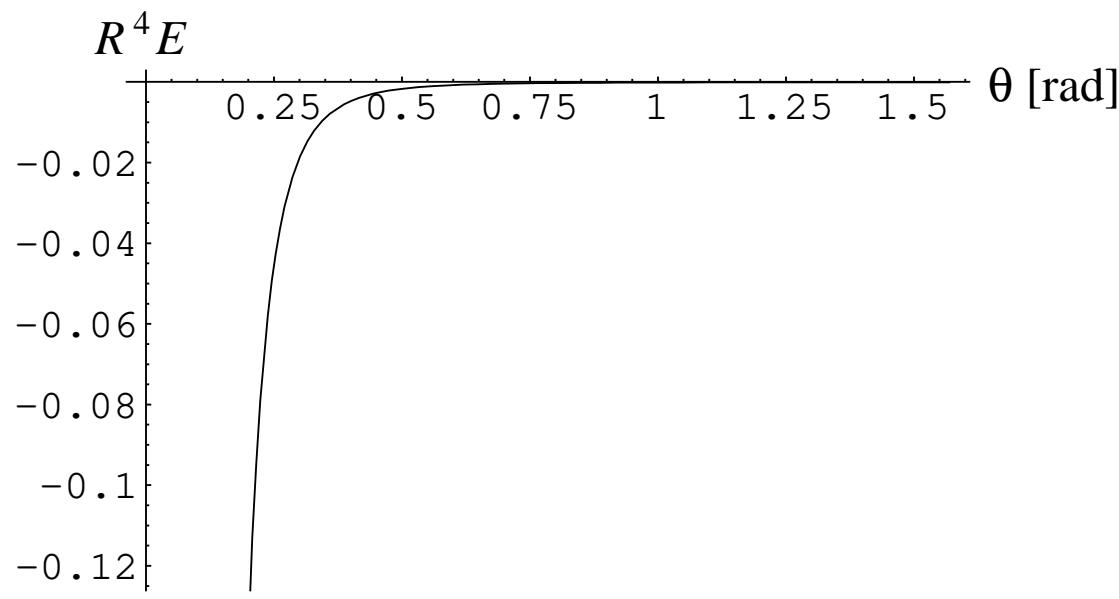

fig. 2: The $\theta$ dependence of the vacuum energy with no boundary condition.

The values are negative for all $\theta$.

The numerical value of $R^{4} E$ which depends on $\theta$ is represented in fig. 2. $R^{4} E$ is negative definite for all $\theta$. 


\section{2. with $\left(\mathbb{Z}_{2}\right)^{2}$ symmetry}

We consider $\left(\mathbb{Z}_{2}\right)^{2}$ symmetry on $T^{2}$. Since the wave function should be an even function of $X_{1}$ and $X_{2}$ respectively on account of the condition (2.6), the eigen function for the mass operator (2.8) becomes proportional to

$$
\cos \left(n_{1} X_{1}\right) \cos \left(n_{2} X_{2}\right)=\cos n_{1}\left(\frac{y_{1}}{R_{1}}-\frac{y_{2}}{R_{1} \tan \theta}\right) \cos n_{2}\left(\frac{y_{2}}{R_{2} \sin \theta}\right), \quad n_{1}, n_{2} \in\{0, \mathbb{N}\}
$$

We then obtain the masses of Kaluza-Klein modes,

$$
M=\frac{1}{R} f_{n_{1}, n_{2}}(\theta), \quad n_{1}, n_{2} \in\{0, \mathbb{N}\}
$$

Note that, since the eigen functions consist of cosine functions, $n_{1}$ and $n_{2}$ are zero or positive integers. In terms of (3.7), we analyse the vacuum energy, and obtain

$$
\begin{aligned}
E_{\mathbb{Z}_{2}}=-\frac{1}{64 \pi^{2}} \sum_{n_{1}, n_{2}=0}^{\infty} M^{4}\left[\ln \frac{\lambda^{2}}{M^{2}}+\frac{3}{2}\right] \\
=\frac{1}{32 \pi^{2} R^{4}} \sum_{n_{1}, n_{2}=0}^{\infty} f_{n_{1}, n_{2}}{ }^{4} \ln f_{n_{1}, n_{2}} \\
=\frac{1}{32 \pi^{2} R^{4}}\left[\frac{1}{2} \sum_{n_{1}=-\infty}^{\infty}\left(1+\delta_{n_{1}, 0}\right)\right]\left[\frac{1}{2} \sum_{n_{2}=-\infty}^{\infty}\left(1+\delta_{n_{2}, 0}\right)\right] f_{n_{1}, n_{2}}{ }^{4} \ln f_{n_{1}, n_{2}} \\
=\frac{1}{128 \pi^{2} R^{4}}\left[\sum_{n_{1}, n_{2}=-\infty}^{\infty} f_{n_{1}, n_{2}}{ }^{4} \ln f_{n_{1}, n_{2}}\right. \\
\left.\quad+\sum_{n_{1}=-\infty}^{\infty} f_{n_{1}, 0}{ }^{4} \ln f_{n_{1}, 0}+\sum_{n_{2}=-\infty}^{\infty} f_{0, n_{2}}{ }^{4} \ln f_{0, n_{2}}\right] .
\end{aligned}
$$

where the last term has been dropped due to the identity $f_{0,0}{ }^{4} \ln f_{0,0}=0$. The first term in (3.8) has already been considered in the previous subsection, and from (3.5) we get

$$
\begin{aligned}
\sum_{n_{1}, n_{2}=-\infty}^{\infty} f_{n_{1}, n_{2}}{ }^{4} \ln f_{n_{1}, n_{2}} & =\left.\sum_{n_{1}, n_{2}=-\infty}^{\infty} \frac{d}{d x} f_{n_{1}, n_{2}}{ }^{x}\right|_{x=4} \\
& =-\frac{1}{2 \pi^{5}} \Gamma(3)^{2} \sin \theta \sum_{\substack{m_{1}, m_{2}=-\infty \\
\left(m_{1}, m_{2}\right) \neq(0,0)}}^{\infty} \frac{1}{\left(m_{1}^{2}+m_{2}^{2} \sin ^{2} \theta\right)^{3}}
\end{aligned}
$$


Using (3.4), we calculate the second term in (3.8) as

$$
\begin{aligned}
\sum_{n_{1}=-\infty}^{\infty} f_{n_{1}, 0}^{4} \ln f_{n_{1}, 0} & =\left.\frac{d}{d x} \sum_{n_{1}=-\infty}^{\infty}\left(\sqrt{n_{1}^{2}}\right)^{x}\right|_{x=4} \\
& =\left.\frac{d}{d x}\left(-\frac{\sin \frac{\pi x}{2}}{\pi^{\frac{3}{2}+4}} \Gamma\left(1+\frac{x}{2}\right) \Gamma\left(\frac{1+x}{2}\right) \sum_{\substack{k=-\infty \\
k \neq 0}}^{\infty}\left(\sqrt{k^{2}}\right)^{-x-1}\right)\right|_{x=4} \\
& =-\frac{1}{2 \pi^{\frac{9}{2}}} \Gamma(3) \Gamma\left(\frac{5}{2}\right) \sum_{\substack{k=-\infty \\
k \neq 0}}^{\infty} \frac{1}{\left(\sqrt{k^{2}}\right)^{5}} \\
& =-\frac{1}{\pi^{\frac{9}{2}}} \Gamma(3) \Gamma\left(\frac{5}{2}\right) \zeta(5) .
\end{aligned}
$$

We can also show in the same way as for (3.10) that the third term in (3.8) becomes

$$
\begin{aligned}
\sum_{n_{2}=-\infty}^{\infty} f_{0, n_{2}}{ }^{4} \ln f_{0, n_{2}} & =\left.\frac{d}{d x} \sum_{n_{2}=-\infty}^{\infty}\left(\sqrt{\frac{n_{2}^{2}}{\sin ^{2} \theta}}\right)^{x}\right|_{x=4} \\
& =\left.\frac{d}{d x}\left(\frac{1}{(\sin \theta)^{x}} \sum_{n_{2}=-\infty}^{\infty}\left(\sqrt{n_{2}^{2}}\right)^{x}\right)\right|_{x=4} \\
& =-\frac{1}{\pi^{\frac{9}{2}} \sin ^{4} \theta} \Gamma(3) \Gamma\left(\frac{5}{2}\right) \zeta(5) .
\end{aligned}
$$

Substituting (3.9), (3.10) and (3.11) into (3.8), the vacuum energy with the condition of $\left(\mathbb{Z}_{2}\right)^{2}$ symmetry is written down as

$$
\begin{aligned}
E_{\mathbb{Z}_{2}}= & -\frac{\Gamma(3)}{256 \pi^{7} R^{4}}\left[\Gamma(3) \sin \theta \sum_{\substack{k, l=-\infty \\
(k, l) \neq(0,0)}} \frac{1}{\left(k^{2}+l^{2} \sin ^{2} \theta\right)^{3}}+2 \sqrt{\pi} \Gamma\left(\frac{5}{2}\right) \zeta(5)\left(1+\frac{1}{\sin ^{4} \theta}\right)\right] \\
= & -\frac{\Gamma(3)}{256 \pi^{7} R^{4}}\left[4 \Gamma(3) \sin \theta \sum_{k, l=1}^{\infty} \frac{1}{\left(k^{2}+l^{2} \sin ^{2} \theta\right)^{3}}\right. \\
& \left.+2 \Gamma(3) \zeta(6)\left(\sin \theta+\frac{1}{\sin ^{5} \theta}\right)+2 \sqrt{\pi} \Gamma\left(\frac{5}{2}\right) \zeta(5)\left(1+\frac{1}{\sin ^{4} \theta}\right)\right]
\end{aligned}
$$




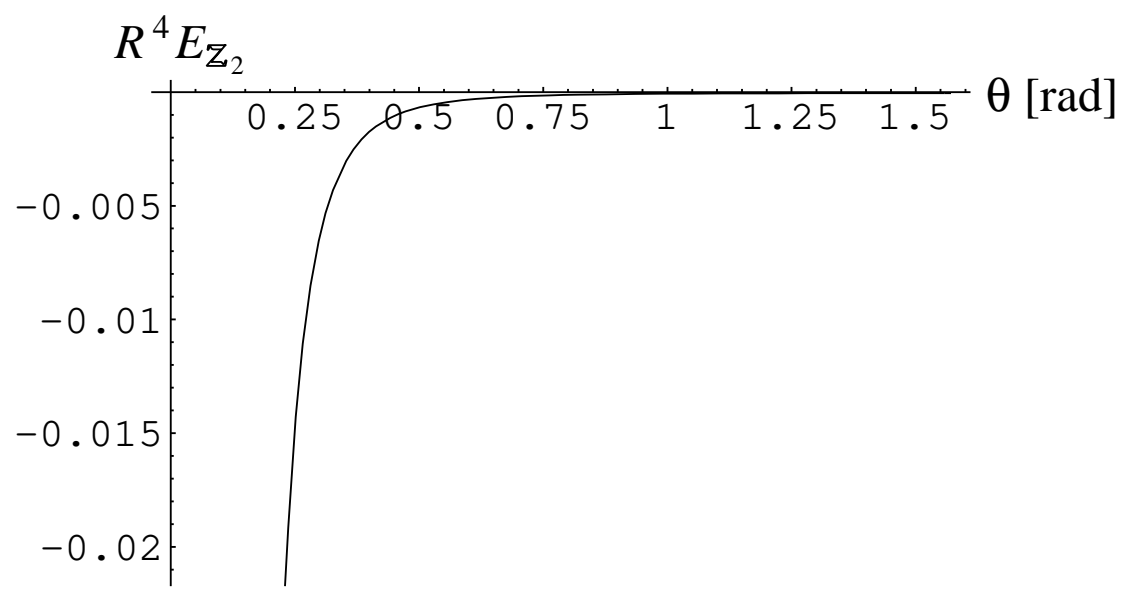

fig. 3: The $\theta$ dependence of the vacuum energy with $\left(\mathbb{Z}_{2}\right)^{2}$ symmetry. The values are negative for all $\theta$.

We can calculate the coefficient of $R^{-4}$ in (3.12) numerically and the result is depicted in fig. 3. The vacuum energy $E_{\mathbb{Z}_{2}}$ is negative for all $\theta$.

\section{3. with Dirichlet condition}

We require the Dirichlet condition on the boundary of the fundamental region of $T^{2}$, that is, on the two lines defined by $y_{1} \tan \theta-y_{2}=0$ and $y_{2}=0$. In other words, the wave function $\psi\left(X_{1}, X_{2}\right)$ should vanish on the lines $X_{1}=0$ and $X_{2}=0$. So the eigen functions of (2.8) are proportional to the products of sine functions,

$$
\sin \left(n_{1} X_{1}\right) \sin \left(n_{2} X_{2}\right)=\sin n_{1}\left(\frac{y_{1}}{R_{1}}-\frac{y_{2}}{R_{1} \tan \theta}\right) \sin n_{2}\left(\frac{y_{2}}{R_{2} \sin \theta}\right), \quad n_{1}, n_{2} \in \mathbb{N} .
$$

The eigen values, that is, the masses of Kaluza-Klein modes become

$$
M=\frac{1}{R} f_{n_{1}, n_{2}}(\theta), \quad n_{1}, n_{2} \in \mathbb{N} .
$$

By the use of (3.9), (3.10) and (3.11), we obtain the vacuum energy,

$$
\begin{aligned}
E_{\text {Dirichlet }} & =-\frac{1}{64 \pi^{2}} \sum_{n_{1}, n_{2}=1}^{\infty} M^{4}\left[\ln \frac{\lambda^{2}}{M^{2}}+\frac{3}{2}\right] \\
& =\frac{1}{32 \pi^{2} R^{4}}\left[\frac{1}{2} \sum_{n_{1}=-\infty}^{\infty}\left(1-\delta_{n_{1}, 0}\right)\right]\left[\frac{1}{2} \sum_{n_{2}=-\infty}^{\infty}\left(1-\delta_{n_{2}, 0}\right)\right] f_{n_{1}, n_{2}}{ }^{4} \ln f_{n_{1}, n_{2}}
\end{aligned}
$$




$$
\begin{aligned}
= & \frac{1}{128 \pi^{2} R^{4}}\left[\sum_{n_{1}, n_{2}=-\infty}^{\infty} f_{n_{1}, n_{2}}{ }^{4} \ln f_{n_{1}, n_{2}}-\sum_{n_{1}=-\infty}^{\infty} f_{n_{1}, 0}{ }^{4} \ln f_{n_{1}, 0}-\sum_{n_{2}=-\infty}^{\infty} f_{0, n_{2}}{ }^{4} \ln f_{0, n_{2}}\right] \\
= & -\frac{\Gamma(3)}{256 \pi^{7} R^{4}}\left[4 \Gamma(3) \sin \theta \sum_{n_{1}, n_{2}=1}^{\infty} \frac{1}{\left(n_{1}^{2}+n_{2}^{2} \sin ^{2} \theta\right)^{3}}\right. \\
& \left.+2 \Gamma(3) \zeta(6)\left(\sin \theta+\frac{1}{\sin ^{5} \theta}\right)-2 \sqrt{\pi} \Gamma\left(\frac{5}{2}\right) \zeta(5)\left(1+\frac{1}{\sin ^{4} \theta}\right)\right] .
\end{aligned}
$$

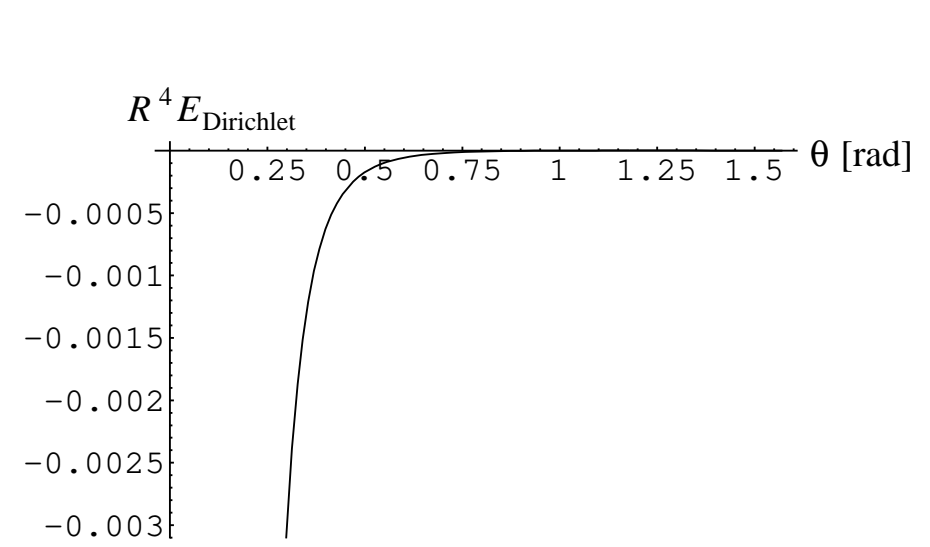

(a)

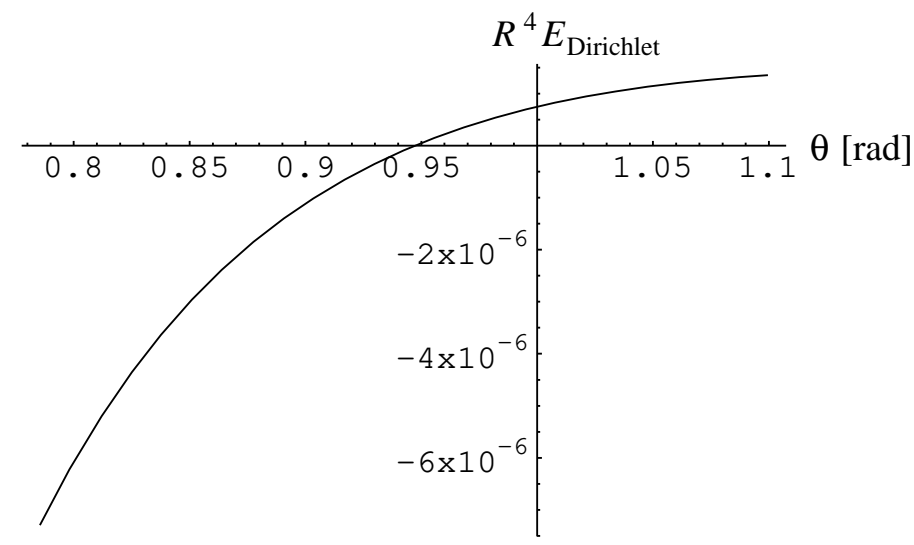

(b)

fig. 4: The $\theta$ dependence of the vacuum energy with Dirichlet condition. The values turn from negative to positive at $\theta=\theta_{\mathrm{cr}}$ as shown in the magnified plot (b).

The results of the numerical calculations of $R^{4} E_{\text {Dirichlet }}$ are represented in fig. 4 . In this case it is significant that the positive vacuum energy appears. The vacuum energy $E_{\text {Dirichlet }}$ is equal to zero at $\theta=\theta_{\text {cr }}$ (fig. $4(\mathrm{~b})$ ). For $\theta<\theta_{\text {cr }}$ the vacuum energy is negative, while for $\theta>\theta_{\mathrm{cr}}$ it is positive. The critical angle $\theta_{\mathrm{cr}}$ is approximately equal to 0.947243 [rad] which is calculated numerically.

The positive vacuum energy is favourable, because the cosmological constant is positive. From this point of view, we will give some comments in the next section.

\section{Comments on Generic Size of Extra Dimensions}

The extra dimensions have been introduced in [1,2] in order to solve the hierarchy problem . When we consider a $4+d$ dimensional space-time, that is, a $d$ dimensional extra 
space, the relation between the Plank scale $M_{\mathrm{pl}}$ of our four dimensional world and the one $M_{\mathrm{pl}(4+d)}$ of the $4+d$ dimensional space-time is described by

$$
M_{\mathrm{pl}}^{2} \sim M_{\mathrm{pl}(4+d)}{ }^{2+d} R^{d}
$$

where $R$ denotes the size of the extra dimensions. When we assume that $M_{\mathrm{pl}(4+d)} \sim$ $m_{\mathrm{EW}} \sim 1[\mathrm{TeV}]$, we obtain the size for the extra space as

$$
R \sim 10^{\frac{30}{d}-17}[\mathrm{~cm}]
$$

For $d=2$, the size $R$ becomes a sub-millimeter size, $10^{-2}[\mathrm{~cm}]$.

On the other hand, we have not had a complete answer for the cosmological constant problem yet. But a lot of features have been made clear by some observations, for a recent example, the WMAP observation. The results of the WMAP 12,13] provide us the following data;

$$
\begin{aligned}
h & =0.71 \quad(\text { Hubble constant }), \\
\Omega_{\text {tot }} & =1.02 \pm 0.02 \quad(\text { total density }), \\
\Omega_{\Lambda} & =0.73 \pm 0.04 \quad \text { (dark energy density). }
\end{aligned}
$$

From (4.1a), the Hubble constant is

$$
H_{0}=h \times 100\left[\mathrm{~km} \cdot \mathrm{sec}^{-1} \cdot \mathrm{Mpc}^{-1}\right]=0.7675 \times 10^{-28}\left[\mathrm{~cm}^{-1}\right]
$$

In terms of (4.2), we obtain the critical density,

$$
\rho_{\mathrm{c}}=\frac{3 H_{0}^{2}}{8 \pi G}=5.313 \times 10^{3}\left[\mathrm{eV} \cdot \mathrm{cm}^{-3}\right]
$$

Since we consider that the dark energy is almost the same as the cosmological constant $\Lambda$, it is calculated from (4.16), (4.10) and (4.3) as

$$
\Lambda=\rho_{\mathrm{c}} \Omega_{\mathrm{tot}} \Omega_{\Lambda}=3.956 \times 10^{3}\left[\mathrm{eV} \cdot \mathrm{cm}^{-3}\right]
$$

Now we assume that the cosmological constant is identified with the vacuum energy to which the Kaluza-Klein modes from the compactification of extra dimensions contribute. The vacuum energy is proportional to $R^{-4}$. If the size of the extra space $R$ is a submillimeter size, $R^{-4}$ is in the order of $10^{8}\left[\mathrm{~cm}^{-4}\right] \sim 10^{4}\left[\mathrm{eV} \cdot \mathrm{cm}^{-3}\right]$. Since it is close to the 
cosmological constant (4.4), that identification is reasonable. The relationship between the vacuum energy, in other words, the Casimir energy, and the cosmological constant has been discussed in the various papers [16,27 37]. We believe from recent observations that the cosmological constant is positive. On account of that identification, the vacuum energy also has to be positive. Fortunately we were able to realize a positive vacuum energy by the use of the extra dimensions with the Dirichlet condition in the section 3.3 .

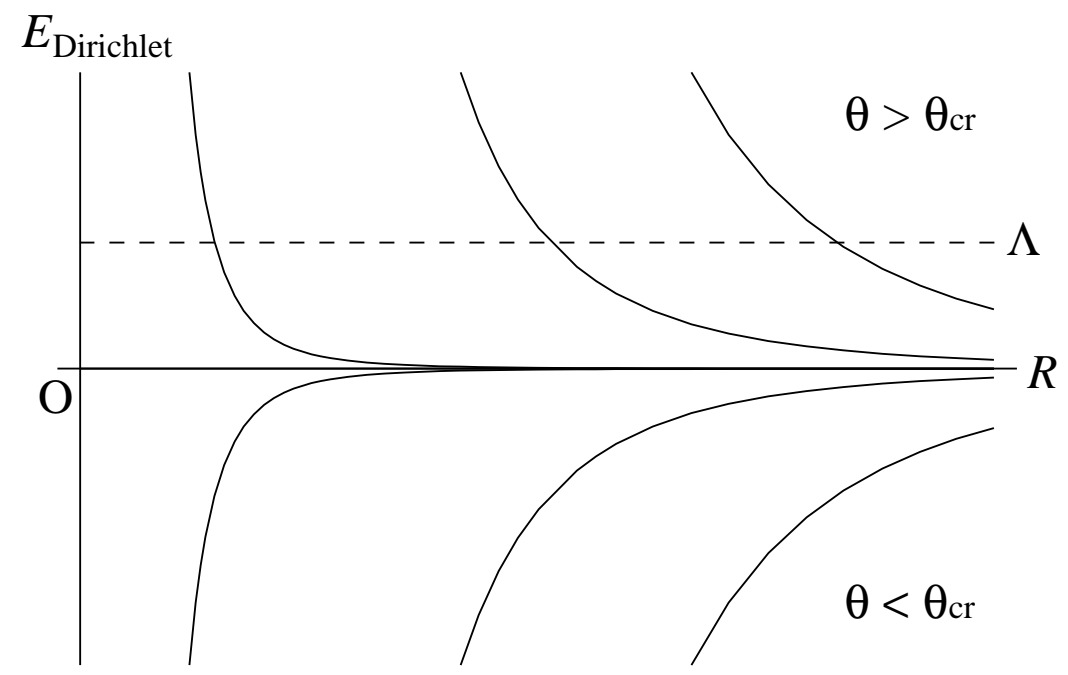

fig. 5: The $R$ dependence of the vacuum energy. If $\left|\theta-\theta_{\mathrm{cr}}\right|$ increases, the corresponding line for the graph of $\left(R, E_{\text {Dirichlet }}\right)$ moves away from the vertical axis.

Since the vacuum energy $E_{\text {Dirichlet }}$ depends on $R^{-4}$ from (3.14), the behaviour of $E_{\text {Dirichlet }}$ for $R$ is represented by fig. 5 .

If the values of the cosmological constant $\Lambda$ and the moduli parameter $\theta$ are given, the size of extra dimensions $R$ is determined. For example, let us consider the case of $\theta=\pi / 2$. This is the situation in which the torus $T^{2}$ has no more shape moduli and is essentially equal to the product of two spheres, $\left(S^{1}\right)^{\otimes 2}$, with the same radius $R$. The vacuum energy (3.14) then becomes $E_{\text {Dirichlet }}=1.1769 \times 10^{-6} / R^{4}$. In terms of $\hbar c=1.973269 \times 10^{-5}[\mathrm{eV} \cdot \mathrm{cm}]$, we obtain

$$
E_{\text {Dirichlet }}\left[\mathrm{eV} \cdot \mathrm{cm}^{-3}\right]=\frac{2.3223 \times 10^{-11}}{(R[\mathrm{~cm}])^{4}} .
$$

When we identify this vacuum energy with the cosmological constant (4.4), the size of the extra dimensions $R$ becomes $2.768 \times 10^{-4}[\mathrm{~cm}]$. Though this scale is different by $10^{-2}$ order from the one derived from the hierarchy problem [1,2], it is not so crucial an inconsistency.

Note that there is no zero mode under the Dirichlet condition. This is a serious problem, if the particle propagating in the extra space is a graviton. But it is not a 
problem for a scalar particle, because, in fact, there is no such a massless scalar particle in our four dimensional space-time.

\section{Discussions and Conclusions}

We considered the torus $T^{2}$ as a two dimensional extra space. We introduced the shape moduli of $T^{2}$ and analysed the dependence of the vacuum energy, or the Casimir energy, on the shape moduli parameter, to which all the Kaluza-Klein modes derived from the $T^{2}$ compactification contributed. Without a condition on the covering space of $T^{2}$ except for bi-periodic condition, we can find eigen functions for the standard mass operator. But, if we require the $\left(\mathbb{Z}_{2}\right)^{2}$ or the Dirichlet conditions, there is no eigen function for the mass operator. So we proposed the new mass operator (2.8) together with the corresponding eigen functions and considered the vacuum energy for this operator.

In terms of the mathematical tools of the Fourier transformation and the generalized functions, we regularized the divergence of the infinite summations of Kaluza-Klein modes. For a specific case of $d=1$, our regularization reduces to the zeta-function regularization, where higher mode is suppressed and infinity arising in infinite volume limit is subtracted. This regularization provides us the same results as are given by the cuttoff method [38]. Our method being generalized to multi-dimensional problems is a valid and unique one to the extent that the zeta-function regularization is a valid method.

We then made it possible to calculate numerically the vacuum energy including the contributions of all the Kaluza-Klein modes. In general the vacuum energy has a negative value. But we have found that, if we impose the Dirichlet conditions, the value of the vacuum energy turns out to be positive lifting from a negative territory, as the shape moduli parameter changes crossing the critical value.

We assumed that the vacuum energy was identified with the cosmological constant. Since it is confirmed by the observations, for example, the WMAP observation, that the cosmological constant is positive, we compared the WMAP's observational value of the cosmological constant with the vacuum energy which we calculated under the Dirichlet condition. From this comparison we showed that the size of extra dimensions is of order $10^{-4}[\mathrm{~cm}]$. The value of $R=2.768 \times 10^{-4}[\mathrm{~cm}]$ presented in the section 4 is the maximal one that we can realize in the model considered in this paper. On the other hand, from the hierarchy problem, the size of extra space for the two extra dimensions is $10^{-2}[\mathrm{~cm}]$. Though these two sizes do not accurately agree with each other, they are sufficiently consistent. 
Since the cosmological constant may consist not only of the vacuum energy but also of some other elements, it is significant that we showed that the two sizes of extra dimensions derived from the hierarchy problem and from the cosmological constant problem are similar.

It is possible to consider various models with an extra space. So one of future problems is for us to find the model in which the vacuum energy has a positive value of right order of magnitude. Since we calculated the vacuum energy only in a semi-classical limit in this paper, it is also a future problem to consider possible quantum loop effects and also some possible contributions coming from quantum fluctuations of relevant metric components.

\section{Acknowledgements}

S.S. is grateful to N. Maru and M. Tachibana for useful discussions. S.M. was supported in part by Grant-in-Aid for Scientific Research on Priority Areas 763 "Dynamics of Strings and Fields," while S.S. by Grant-in-Aid for the 21st Century COE "Center for Diversity and Universality in Physics," from the Ministry of Education, Culture, Sports, Science and Technology, Japan. 


\section{References}

[1] N. Arkani-Hamed, S. Dimopoulos and G. Dvali, "The Hierarchy Problem and New Dimensions at a Millimeter," Phys. Lett. B429 (1998) 263, hep-ph/9803315.

[2] I. Antoniadis, N. Arkani-Hamed, S. Dimopoulos and G. Dvali, "New Dimensions at a Millimeter to a Fermi and Superstrings at a TeV," Phys. Lett. B436 (1998) 257, hep-ph/9804398.

[3] L. Randall and R. Sundrum, "A Large Mass Hierarchy from a Small Extra Dimension," Phys. Rev. Lett. 83 (1999) 3370, hep-ph/9905221.

[4] L. Randall and R. Sundrum, "An Alternative to Compactification," Phys. Rev. Lett. 83 (1999) 4690, hep-th/9906064.

[5] N. Arkani-Hamed, S. Dimopoulos and J. March-Russell, "Stabilization of Submillimeter Dimensions: The New Guise of the Hierarchy Problem," Phys. Rev. D63 (2001) 064020, hep-th/9809124.

[6] S. Nojiri, S. D. Odintsov and S. Zerbini, "Bulk versus Boundary (Gravitational Casimir) Effects in Quantum Creation of Inflationary Brane-world Universe," Class. Quant. Grav. 17 (2000) 4855, hep-th/0006115.

[7] D. Cremades, L. E. Ibanez and F. Marchesano, "Standard Model at Intersecting D5branes: Lowering the String Scale," Nucl. Phys. B643 (2002) 93, hep-th/0205074.

[8] C. Kokorelis, "Exact Standard Model Structures from Intersecting D5-Branes," Nucl. Phys. B677 (2004) 115, hep-th/0207234.

[9] G. Cognola, E. Elizalde, S. Nojiri, S. D. Odintsov and S. Zerbini, "Multi-graviton Theory from a Discretized RS Brane-world and the Induced Cosmological Constant," hep-th/0312269.

[10] A. G. Riess et al., "Observational Evidence from Supernovae for an Accelerating Universe and a Cosmological Constant," Astron. J. 116 (1998) 1009, astro-ph/9805201.

[11] S. Perlmutter et al., "Measurements of Omega and Lambda from 42 High-Redshift Supernovae," Astrophys. J. 517 (1999) 565, astro-ph/9812133.

[12] C. L. Bennett et al., "First Year Wilkinson Microwave Anisotropy Probe (WMAP) Observations: Preliminary Maps and Basic Results," Astrophys. J. Suppl. 148 (2003) 1, astro-ph/0302207; see also the Web page, http://map.gsfc.nasa.gov/.

[13] D. N. Spergel et al., "First Year Wilkinson Microwave Anisotropy Probe (WMAP) Observations: Determination of Cosmological Parameters," Astrophys. J. Suppl. 148 (2003) 175, astro-ph/0302209.

[14] M. Fukuma, Y. Kono and A. Miwa, "A Mechanism of the Large-scale Damping in the CMB Anisotropy," hep-th/0312298.

[15] M. Fukuma, Y. Kono and A. Miwa, "Noncommutative Inflation and the Large-scale Damping in the CMB Anisotropy," hep-th/0401153. 
[16] S. Matsuda and S. Seki, "Proposal for Generic Size of Large Extra Dimensions," hep$\mathrm{ph} / 0307361$.

[17] N. Kaloper, J. March-Russell, G. D. Starkman and M. Trodden, "Compact Hyperbolic Extra Dimensions: Branes, Kaluza-Klein Modes and Cosmology," Phys. Rev. Lett. 85 (2000) 928, hep-ph/0002001.

[18] K. R. Dienes, "Shape versus Volume: Making Large Flat Extra Dimensions Invisible," Phys. Rev. Lett. 88 (2002) 011601, hep-ph/0108115.

[19] K. R. Dienes and A. Mafi, "Shadows of the Planck Scale: The Changing Face of Compactification Geometry," Phys. Rev. Lett. 88 (2002) 111602, hep-th/0111264.

[20] K. R. Dienes, "Beautified with Goodly Shape: Rethinking the Properties of Large Extra Dimensions," proceedings of the SUSY 2002 Conference, hep-ph/0211211.

[21] E. Ponton and E. Poppitz, "Casimir Energy and Radius Stabilization in Five and Six Dimensional Orbifolds," JHEP 0106 (2001) 019, hep-ph/0105021.

[22] M. Byrne and C. Kolda, "Quintessence and Varying $\alpha$ from Shape Moduli," hepph/0402075.

[23] T. Han, J. D. Lykken and R.-J. Zhang, "On Kaluza-Klein States from Large Extra Dimensions," Phys. Rev. D59 (1999) 105006, hep-ph/9811350.

[24] J. Ambjørn and S. Wolfram, "Properties of the Vacuum 1. Mechanical and Thermodynamic," Annals Phys. 147 (1983) 1.

[25] J. Ambjørn and S. Wolfram, "Properties of the Vacuum 2. Electrodynamic," Annals Phys. 147 (1983) 33.

[26] I. M. Gel'fand and G. E. Shilov, "Generalized Functions," Volume 1 (Academic Press, 1964).

[27] M. D. Roberts, "Vacuum Energy," hep-th/0012062.

[28] K. R. Dienes, "Solving the Hierarchy Problem without Supersymmetry or Extra Dimensions: An Alternative Approach," Nucl. Phys. B611 (2001) 146, hep-ph/0104274.

[29] M. Peloso and E. Poppitz, "Quintessence from Shape Moduli," Phys. Rev. D68 (2003) 125009, hep-ph/0307379.

[30] C. L. Gardner, "Primordial Inflation and Present-Day Cosmological Constant from Extra Dimensions," Phys. Lett. B524 (2002) 21, hep-th/0105295.

[31] K. A. Milton, "Constraints on Extra Dimensions from Cosmological and Terrestrial Measurements," Grav. Cosmol. 8 (2002) 65, hep-th/0107241.

[32] I. O. Cherednikov, "On Casimir Energy Contribution to Observable Value of the Cosmological Constant," astro-ph/0111287.

[33] A. C. Melissinos, "Vacuum Energy and the Cosmological Constant," hep-ph/0112266.

[34] A. Gupta, "Contribution of Kaluza-Klein Modes to Vacuum Energy in Models with Large Extra Dimensions \& the Cosmological Constant," hep-th/0210069.

[35] K. A. Milton, "Dark Energy as Evidence for Extra Dimensions," Grav. Cosmol. 9 (2003) 66, hep-ph/0210170. 
[36] F. Bauer, M. Lindner and G. Seidl, "Casimir Energy in Deconstruction and the Cosmological Constant," hep-th/0309200.

[37] E. Elizalde, "Cosmological Uses of Casimir Energy," hep-th/0311195.

[38] T. Appelquist and A. Chodos, "Quantum Dynamics of Kaluza-Klein Theories," Phys. Rev. D28 (1983) 772. 